\title{
Every reasonably sized matrix group is a subgroup of $S_{\infty}$
}

\author{
by
}

\author{
Robert R. Kallman (Denton, TX)
}

\begin{abstract}
Every reasonably sized matrix group has an injective homomorphism into the group $S_{\infty}$ of all bijections of the natural numbers. However, not every reasonably sized simple group has an injective homomorphism into $S_{\infty}$.
\end{abstract}

1. Introduction. If $X$ is any nonempty set, let $S(X)$ be the set of bijections of $X$. Let $S_{\infty}=S(\mathbb{N})$, where $\mathbb{N}$ is the set of natural numbers. Of course, we may think of $S_{\infty}$ as the set of all permutations of any countable set. It is natural to ask: to what extent is Cayley's Theorem true for $S_{\infty}$ ?

In 1960 S. M. Ulam ([11] and [12], page 58) asked "Can one show that the group $R$ of all rotations in the three-dimensional space is isomorphic (as an abstract group, not continuously, of course) to a subgroup of the group $S_{\infty}$ of all permutations of integers? Or, perhaps quite generally: is every Lie group isomorphic (as an abstract group) to a subgroup of the group $S_{\infty}$ ?" These questions are obviously motivated by Problem 95 of the Scottish Book ([8]), due to Schreirer and Ulam (November 1935), who asked and answered this question for $(\mathbb{R},+)$.

The purpose of this paper is to prove the following theorem. It certainly answers Ulam's first question and a large portion of the second.

THEOREM 1. Let $n$ be a positive integer and $F$ be a field of arbitrary characteristic such that $\operatorname{card}(F) \leq 2^{\aleph_{0}}$. Then there is an injective homomorphism of $\mathrm{GL}(n, F)$ into $S_{\infty}$.

The proof is rather elementary, requiring what at best are minor perturbations of well known results in field theory. Note that since $\mathbb{N}$ can be decomposed into a countable number of countable subsets, the product of

2000 Mathematics Subject Classification: 20B30, 20H20, 12J25, 12F99, 22 A05.

Key words and phrases: infinite symmetric group, matrix groups, nonarchimedian absolute values, field extensions, topological groups. 
countably many copies of $S_{\infty}$ can be embedded into $S_{\infty}$. This implies the following corollary.

Corollary 2. Let $G=\prod_{k \geq 1} G_{k}$, where each $G_{k}$ is one of the groups described in Theorem 1 or $G_{k}=\{e\}$. Then there is an injective homomorphism of $G$ into $S_{\infty}$.

Recall that the rotation group in three dimensions $\mathrm{SO}(3)$ has no subgroups of finite index. A simple consequence of Theorem 1 is that $\mathrm{SO}(3)$ does have subgroups of countable index.

It is not clear just what groups have an injective homomorphism into $S_{\infty}$. However, the following theorem proves that not all reasonably sized groups have an injective homomorphism into $S_{\infty}$.

THEOREM 3. There exists a simple group $G$ such that $\operatorname{card}(G)=2^{\aleph_{0}}$ and there is no injective homomorphism of $G$ into $S_{\infty}$.

The group $G$ of Theorem 3 can be taken to be $S_{\infty} / S_{\mathrm{f}}$, where $S_{\mathrm{f}}$ is the normal subgroup of $S_{\infty}$ consisting of all permutations which move only finitely many integers. Theorem 3 should be compared with the results of de Bruijn [3] who proves, for example, that $S_{\infty}$ can be embedded into $S_{\infty} / S_{\mathrm{f}}$.

2. Proof of Theorem 1. The bulk of the proof will be carried out in a sequence of simple and probably well known lemmas.

LEMma 4. Let $F_{1}$ and $F_{2}$ be two fields which have the same characteristic, are algebraically closed, and satisfy $\operatorname{card}\left(F_{1}\right)=\operatorname{card}\left(F_{2}\right)>\aleph_{0}$. Then $F_{1}$ and $F_{2}$ are isomorphic fields.

Proof. Let $P_{j}$ be the prime subfield of $F_{j}(j=1,2) . P_{1}$ is isomorphic to $P_{2}$ since $F_{1}$ and $F_{2}$ have the same characteristic. Let $B_{j}$ be a transcendence basis for $F_{j}$ over $P_{j}$, so that $F_{j}$ is the algebraic closure of $P_{j}\left(B_{j}\right)$. Since $F_{j}$ is uncountable so is $P_{j}\left(B_{j}\right)$ and hence $B_{j}$ is infinite. Thus $\operatorname{card}\left(P_{j}\left(B_{j}\right)\right)=\operatorname{card}\left(B_{j}\right)$ and $\operatorname{card}\left(P_{j}\left(B_{j}\right)\right)=\operatorname{card}\left(F_{j}\right)$ (Kaplansky [7], Theorem 65, p. 74$)$. It follows that $\operatorname{card}\left(B_{1}\right)=\operatorname{card}\left(B_{2}\right)$. Hence, $P_{1}\left(B_{1}\right)$ and $P_{2}\left(B_{2}\right)$ are isomorphic fields and so are their algebraic closures $F_{1}$ and $F_{2}$.

COROLlary 5. Let $F$ be a field which is algebraically closed and satisfies $\operatorname{card}(F)=2^{\aleph_{0}}$. If $F$ has characteristic zero, then $F$ is algebraically isomorphic to the algebraic closure of the q-adic numbers $\mathbb{Q}_{q}$ for any prime $q$ or to the field of complex numbers $\mathbb{C}$. If $F$ has characteristic $p$, then $F$ is algebraically isomorphic to the algebraic closure of the field of formal Laurent series $F_{p}((x))$, where $F_{p}$ is the field of $p$ elements.

Proof. We have

$$
\operatorname{card}\left(\mathbb{Q}_{q}\right)=2^{\aleph_{0}} \quad \text { and } \quad \operatorname{card}\left(F_{p}((x))\right)=2^{\aleph_{0}} .
$$

Now use Lemma 4 and Kaplansky [7], Theorem 65, p. 74. 
Recall the elementary fact that if $F$ is a field, $|\cdot|$ is a nonarchimedian absolute value on $F, x, y \in F$, and $|y|<|x|$, then $|x+y|=|x|$ (Artin [1], Corollary 5.1).

Lemma 6. Let $L$ be a field, $K \subset L$ a subfield such that $L$ is algebraic over $K,|\cdot|$ a nonarchimedian absolute value on $L$, and $d(u, v)=|u-v|$ for all $u, v \in L$. Suppose that $(K, d)$ is a separable metric space. Then $(L, d)$ is a separable metric space.

Proof. The key idea in this proof can be found in Artin [1], p. 45.

We can assume that $L$ is algebraically closed, for $|\cdot|$ extends to be a nonarchimedian absolute value on the algebraic closure of $K$ (Bourbaki [2], Proposition 9, p. 428).

Let $F$ be a countable subfield of $K$ which is $d$-dense in $K$. It suffices to prove that the roots of the monic polynomials in $F[x]$ are dense in $L$. Let $u \in L$, let $f(x)=x^{n}+a_{n-1} x^{n-1}+\ldots+a_{1} x+a_{0} \in K[x]$ be the irreducible polynomial for $u$ over $K$, let $C=\max \left\{1,\left|a_{n-1}\right|, \ldots,\left|a_{1}\right|,\left|a_{0}\right|\right\}$, and let $\varepsilon>0$. Note that if $v \in L$ with $|v|>C \geq 1$ and if $0 \leq j \leq n-1$, then $\left|a_{j} v^{j}\right| \leq$ $C|v|^{j}<|v|^{n}$, hence $\left|a_{0}+a_{1} v+\ldots+a_{n-1} v^{n-1}\right| \leq \max _{0 \leq j \leq n-1}\left|a_{j} v^{j}\right|<|v|^{n}$, and therefore $|f(v)|=|v|^{n}>0$. Since $u$ is a root of $f(x),|u| \leq C$. Choose $b_{0}, b_{1}, \ldots, b_{n-1} \in F$ such that $\max _{0 \leq j \leq n-1}\left|a_{j}-b_{j}\right|<\varepsilon$. Let $g(x)=x^{n}+$ $b_{n-1} x^{n-1}+\ldots+b_{1} x+b_{0} \in F[x]$. We have $g(x)=\left(x-v_{1}\right) \ldots\left(x-v_{n}\right)$, where $v_{1}, \ldots, v_{n} \in L$. Then $\left|\left(u-v_{1}\right) \ldots\left(u-v_{n}\right)\right|=|g(u)|=|f(u)-g(u)| \leq$ $\max _{0 \leq j \leq n-1}\left|\left(a_{j}-b_{j}\right) u^{j}\right|<\varepsilon C^{n}$. Hence, there exists at least one $1 \leq k \leq n$ such that $\left|u-v_{k}\right| \leq C \sqrt[n]{\varepsilon}$.

Lemma 7. Let $F$ be a field which satisfies $\operatorname{card}(F) \leq 2^{\aleph_{0}}$. Then there is a nonarchimedian absolute value $|\cdot|$ on $F$ with countable range and under which $F$ becomes a separable metric space. If $\operatorname{card}(F)>\aleph_{0}$, then $|\cdot|$ is a nontrivial absolute value.

Proof. That the range of $|\cdot|$ is countable is not essential for the proof of Theorem 1, but does seem to be of independent interest.

We may assume that $F$ is algebraically closed and that $\operatorname{card}(F)=2^{\aleph_{0}}$. If not, enlarge $F$ to $F(B)$, where $B$ is a set of transcendental elements so that $\operatorname{card}(F(B))=2^{\aleph_{0}}$, and let $K$ be the algebraic closure of $F(B)$. Then $\operatorname{card}(K)=\operatorname{card}(F(B))=2^{\aleph_{0}}$ (Kaplansky [7], Theorem 65, p. 74) and replace $F$ with $K$.

If $F$ has characteristic 0 , let $q$ be a fixed prime. Corollary 5 implies that we may assume that $F$ is the algebraic closure of $\mathbb{Q}_{q}$, which is complete under $|\cdot|$. The $q$-adic absolute value $|\cdot|_{q}$ extends in a unique manner to be an absolute value $|\cdot|$ on $F$ (Bourbaki [2], Proposition 10, p. 429). The construction of $|\cdot|$ shows that the range of $|\cdot|$ is countable since the range of $|\cdot|_{q}$ is countable. Lemma 6 implies that $(F, d)$ is a separable metric space 
since $\left(\mathbb{Q}_{q}, d\right)$ is a separable metric space $\left(\mathbb{Q}\right.$ is dense in $\left.\mathbb{Q}_{q}\right)$ and $F$ is an algebraic extension of $\mathbb{Q}_{q}$.

Next, suppose that $F$ has characteristic $p$. In this case Corollary 5 implies that we may assume that $F$ is the algebraic closure of the field of formal Laurent series $F_{p}((x))$, where $F_{p}$ is the field of $p$ elements. $F_{p}((x))$ is complete under a natural absolute value $|\cdot|$ (Jacobson $[6]$, Theorem 9.16, p. 577). As in the characteristic 0 case, the absolute value $|\cdot|$ on the field $F_{p}((x))$ extends in a unique manner to be an absolute value, also denoted by $|\cdot|$, on $F$. The construction of $|\cdot|$ again shows that the range of $|\cdot|$ is countable. Lemma 6 again implies that $(F, d)$ is a separable metric space since $F_{p}((x))$ is a separable metric space (the finite Laurent series with coefficients in $F_{p}$ are dense in $\left.F_{p}((x))\right)$ and $F$ is an algebraic extension of $F_{p}((x))$.

Finally, if $\operatorname{card}(F)>\aleph_{0}$, then the nonarchimedian absolute value $|\cdot|$ is nontrivial since $(F, d)$ is a separable metric space.

The argument given in the next lemma is inspired by that sketched in Serre [10], page LG 4.4, in case $F$ is locally compact.

LemMa 8. Let $F$ be a field, $|\cdot|$ a nontrivial nonarchimedian absolute value on $F$ under which $F$ is a separable metric space, $A=[a \in F|| a \mid \leq 1]$, and $n \geq 2$. Then $\mathrm{SL}(n, A)$ is a proper subgroup of $\mathrm{SL}(n, F)$ of index $\leq \aleph_{0}$.

Proof. Identify $\operatorname{SL}(n, F)$ with a subset of $F^{n^{2}}$ by concatenating the rows of each element of $\mathrm{SL}(n, F)$ and give $\mathrm{SL}(n, F)$ the relative topology. $\mathrm{SL}(n, F)$ then certainly is a separable metric topological group.

Next, recall the elementary facts that $A$ is a commutative ring with identity since $|\cdot|$ is a nonarchimedian absolute value and that $A$ is open in $F$, for if $a \in A$ and $b \in F$ satisfies $|b|<1$, then $|a+b| \leq \max (|a|,|b|) \leq 1$, and therefore the ball $B(a, 1) \subset A$. Next, $A^{n^{2}}$ is an open subset of $F^{n^{2}}$ and thus $\operatorname{SL}(n, A)=\operatorname{SL}(n, F) \cap A^{n^{2}}$ is an open subset of $\operatorname{SL}(n, F)$. Note that $\mathrm{SL}(n, A)$ is closed under multiplication since $A$ is a ring. $\operatorname{SL}(n, A)$ is also closed under inversion by using Cramer's Rule, again since $A$ is a ring. Hence, $\operatorname{SL}(n, A)$ is an open subgroup of $\operatorname{SL}(n, F)$. Further, $\operatorname{SL}(n, A)$ is a proper subgroup of $\mathrm{SL}(n, F)$ since $|\cdot|$ is a nontrivial absolute value.

The quotient topological space $\operatorname{SL}(n, F) / \operatorname{SL}(n, A)$ is therefore discrete and separable and not just a single point. Hence,

$$
1<\operatorname{card}(\mathrm{SL}(n, F) / \mathrm{SL}(n, A)) \leq \aleph_{0} .
$$

Corollary 9. Use the notation of Lemma 8. Let $\varphi: \operatorname{SL}(n, F) \rightarrow$ $S(\mathrm{SL}(n, F) / \mathrm{SL}(n, A))$ be the natural homomorphism. Then the kernel of $\varphi$ is $Z(\mathrm{SL}(n, F))$, the center of $\mathrm{SL}(n, F)$. In particular, there is an injective homomorphism of $\mathrm{SL}(n, F) / Z(\mathrm{SL}(n, F))$ into $S_{\infty}$. 
Proof. If $x \in \operatorname{SL}(n, F)$, then $\varphi(x)$ is the identity if and only if $x \in$ $\bigcap_{y \in \mathrm{SL}(n, F)} y \mathrm{SL}(n, A) y^{-1}=N$, a normal subgroup of $\operatorname{SL}(n, F)$. We infer that $N$ is a proper subgroup of $\operatorname{SL}(n, F)$ by Lemma 8 . Hence, $x \in$ $Z(\mathrm{SL}(n, F))$ by Dieudonné [4], pp. 38-39. On the other hand, $Z(\mathrm{SL}(n, F)) \subset$ $\mathrm{SL}(n, A)$ since $Z(\mathrm{SL}(n, F))$ consists of diagonal matrices.

Corollary 10. Use the notation of Lemma 8. Let $G \subset \mathrm{SL}(n, F)$ be a subgroup such that $G \cap Z(\mathrm{SL}(n, F))=\{e\}$. Then there is an injective homomorphism of $G$ into $S_{\infty}$.

P r o of. There is an injective homomorphism of $G$ into the quotient group $\mathrm{SL}(n, F) / Z(\mathrm{SL}(n, F))$. Now use Corollary 9 .

We are now set to complete the proof of Theorem 1 . We can assume that $\operatorname{card}(F)=2^{\aleph_{0}}$ and that $F$ is algebraically closed by the proof of Lemma 7 . The same lemma implies that there is a nontrivial nonarchimedian absolute value $|\cdot|$ on $F$ under which $F$ is a separable metric space. Define an injective homomorphism $\varphi: \mathrm{GL}(n, F) \rightarrow \mathrm{SL}(n+2, F)$ as follows: if $x \in \mathrm{GL}(n, F)$, let $\varphi(x)_{i, j}=x_{i, j}$ for $1 \leq i, j \leq n$,

$$
\varphi(x)_{i, j}= \begin{cases}\operatorname{det}(x)^{-1} & \text { for } i=j=n+1 \\ \varphi(x)_{i j}=1 & \text { for } i=j=n+2 \\ \varphi(x)_{i j}=0 & \text { otherwise. }\end{cases}
$$

Then $\varphi(\mathrm{GL}(n, F)) \cap Z(\mathrm{SL}(n+2, F))=\{e\}$ since $Z(\mathrm{SL}(n+2, F))$ consists of scalar multiples of the identity matrix. Hence, Corollary 10 implies that there is an injective homomorphism of $\varphi(\mathrm{GL}(n, F))$, and therefore of $\mathrm{GL}(n, F)$, into $S_{\infty}$.

3. Proof of Theorem 3. If $F \subset \mathbb{N}$ is a nonempty finite set, define $U(F)=\left[\pi \in S_{\infty} \mid \pi(x)=x\right.$ for every $\left.x \in F\right]$. Each $U(F)$ is a subgroup of $S_{\infty}$ of countable index. There is a unique Hausdorff topological group topology on $S_{\infty}$ such that the $U(F)$ 's form a basis for the topology of $S_{\infty}$ at the identity. It is simple to check that $S_{\infty}$ is a complete separable metric topological group in this topology.

LEMMA 11. Let $H$ be a topological group such that every subgroup of at most countable index is open and let $K$ be a topological group such that the open subgroups of at most countable index form a basis at $e$ in $K$. Then every group homomorphism $\varphi: H \rightarrow K$ is continuous.

Proof. Let $U$ be an open subgroup of $K$ of at most countable index. Then $\varphi^{-1}(U)$ is a subgroup of $H$ which is of at most countable index and therefore is open. Since such $U$ 's form a basis at $e$ in $K, \varphi$ is continuous at $e$ in $H$, and therefore $\varphi$ is continuous.

Corollary 12. Every group homomorphism $\psi: S_{\infty} \rightarrow S_{\infty}$ is continuous. 
Proof. The open subgroups of countable index form a basis for the topology of $S_{\infty}$ at $e$. On the other hand, subgroups of countable index in $S_{\infty}$ are open by Dixon et al. [5], Theorem 1, p. 580. Now use Lemma 11.

We are now ready to complete the proof of Theorem 3 . Let $S_{\mathrm{f}}$ be the normal subgroup of $S_{\infty}$ consisting of all permutations which move only finitely many integers. $S_{\infty} / S_{\mathrm{f}}$ is known to be a simple group (Schreier and Ulam [9], Satz 1, p. 135). Suppose that $\varphi: S_{\infty} / S_{\mathrm{f}} \rightarrow S_{\infty}$ is an injective homomorphism. Let $\pi: S_{\infty} \rightarrow S_{\infty} / S_{\mathrm{f}}$ be the natural surjective quotient mapping and let $\psi=\varphi \circ \pi$. Then $\psi$ is a group homomorphism. $\psi$ is continuous by Lemma 12. But $S_{\mathrm{f}}$ is in the kernel of $\psi$ and $S_{\mathrm{f}}$ is dense in $S_{\infty}$. Hence $\psi$ is trivial and therefore $\varphi$ is trivial. Contradiction. So there is no injective homomorphism of $S_{\infty} / S_{\mathrm{f}}$ into $S_{\infty}$.

4. Remark. The referee has pointed out that the separability of the space $(F, d)$ and the countability of the index of the subgroup $\operatorname{SL}(n, A)$ in $\mathrm{SL}(n, F)$ can be proved algebraically in the special cases $F=\overline{\mathbb{Q}}_{p}$ and $F=$ $\bar{F}_{p}((x))$, which allows for an alternative purely algebraic proof of Theorem 1.

\section{References}

[1] E. Artin, Algebraic Numbers and Algebraic Functions, Gordon and Breach, New York, 1967.

[2] N. Bourbaki, Commutative Algebra, Addison-Wesley, Reading, MA, 1972.

[3] N. G. de Bruijn, Embedding theorems for infinite groups, Indag. Math. 19 (1957), 560-569; Konink. Nederl. Akad. Wetensch. Proc. 60 (1957), 560-569.

[4] J. Dieudonné, La géométrie des groupes classiques, 2nd ed., Springer, Berlin, 1963.

[5] J. D. Dixon, P. M. Neumann and S. Thomas, Subgroups of small index in infinite symmetric groups, Bull. London Math. Soc. 18 (1986), 580-586.

[6] N. Jacobson, Basic Algebra II, W. H. Freeman, San Francisco, 1980.

[7] I. Kaplansky, Fields and Rings, 2nd ed., Univ. of Chicago Press, Chicago, 1973.

[8] R. D. Mauldin (ed.), The Scottish Book, Birkhäuser, Boston, 1981.

[9] J. Schreier und S. M. Ulam, Über die Permutationsgruppe der natürlichen Zahlenfolge, Studia Math. 4 (1933), 134-141.

[10] J.-P. Serre, Lie Algebras and Lie Groups, W. A. Benjamin, New York, 1965.

[11] S. M. Ulam, A Collection of Mathematical Problems, Wiley, New York, 1960.

[12] - Problems in Modern Mathematics, Wiley, New York, 1964.

Department of Mathematics

University of North Texas

P.O. Box 311430

Denton, TX 76203-1430, U.S.A.

E-mail: fe60@unt.edu

Received 8 January 1999;

in revised form 9 February 2000 and 11 March 2000 